\title{
Highest Level of Support
}

National Cancer Institute

\section{Source}

National Cancer Institute. Highest Level of Support. NCI Thesaurus. Code C104608.

An answer confirming the highest level of support. 